\title{
Dermatological Manifestations in People with Obesity
}

\author{
Venna Ashok Babu' ${ }^{1}$ Gulam Mohamed Shahul Hameed ${ }^{2}$ \\ ${ }^{1}$ Department of DVL, Mamata General Hospital, Khammam, Telangana, India. \\ ${ }^{2}$ Department of DVL, Sri Muttu Kumaran Medical College, Chennai, Tamilnadu, India.
}

\section{ABSTRACT}

\section{BACKGROUND}

Obesity is recognized worldwide as a major health problem. Obesity is defined as a body mass index (BMI) of $30 \mathrm{Kg} / \mathrm{m}^{2}$ or greater. Obesity is associated with an increased risk of health problems including hypertension, type-2 diabetes mellitus, dyslipidemias along with skin conditions such as acrochordons, acanthosis nigricans, striae distensae and pyodermas. which can be easily diagnosed on inspection.

\section{METHODS}

The study was carried out from January 2018 to January 2019. This is a cross sectional descriptive study. The study comprised of 50 adult patients ( 25 males, 25 females) of age more than 15 years satisfying the inclusion criteria. Serum lipid profiles, fasting blood sugar, post prandial blood sugar, fasting serum insulin, fasting serum leptin, liver function tests, renal function tests and clinical examination were done to exclude hypertension and cardiovascular diseases. Chi-square and Fisher exact tests were used to test the significant associations.

\section{RESULTS}

Majority ( $>40 \%$ ) of patients were in third and fourth decades of life. Significantly more skin diseases were found in obese patients. Acanthosis nigricans, skin tags, tinea corporis, intertrigo, striae distensae, psoriasis, fissure feet, pyodermas, varicose veins, seborrhoeic dermatitis, were significantly seen. Many patients had more than one skin condition. Acanthosis nigricans and skin tags were the most prevalent skin changes. Diabetes mellitus was the most commonly seen systemic illness.

\section{CONCLUSIONS}

Dermatologists need to be aware of skin diseases related to obesity so that they can advise the patients regarding early monitoring and associated risk factors like diabetes, dyslipidemias and cardiovascular diseases.

\section{KEY WORDS}

Obesity, Acanthosis Nigricans, Acrochordons, Striae Distensae
Corresponding Author: Dr. Venna Ashok Babu, H. No. 11-2-80/1,

Balaji Nagar, Wyra Road, Khammam-507001, Telangana, India. E-mail: drashok24@gmail.com

DOI: $10.14260 /$ jemds/2019/767

Financial or Other Competing Interests: None.

How to Cite This Article:

Babu VA, Hameed GMS. Dermatological manifestations in people with obesity. J. Evolution Med. Dent. Sci. 2019;8(47):35493553, DOI: 10.14260/jemds/2019/767

Submission 21-09-2019,

Peer Review 08-11-2019,

Acceptance 15-11-2019,

Published 25-11-2019. 


\section{BACKGROUND}

Obesity is a state of excess adipose tissue mass. The most widely used method to gauge obesity is the body mass index (BMI), which is equal to weight/height ${ }^{2}$ (in $\left.\mathrm{Kg} / \mathrm{m}^{2}\right) .{ }^{1}$ Based on the substantial delay of morbidity a BMI of 30 is most commonly used as a threshold for obesity in both men and women. A waist hip ratio of $>0.9$ in women and $>1.0$ in men are abnormal.

\begin{tabular}{|c|c|c|}
\hline WHO Classification & BMI (Kg/ $\left.\mathbf{m}^{\mathbf{2}}\right)$ & Risk of Comorbidities \\
\hline Overweight & $25-30$ & Mildly increased \\
\hline Obese & $>30$ & Moderate \\
\hline Class I & $30-35$ & Severe \\
\hline Class II & $35-40$ & Very Severe \\
\hline Class III & $>40$ & \multicolumn{2}{|c|}{} \\
\hline Range of Body Mass Index Used to Classify Degrees of Over Height and \\
Associated Risk of Comorbidities.
\end{tabular}

\section{Pathophysiology of Obesity}

Obesity results from both environmental and genetic factors. Based on previous studies, approximately $60 \%$ to $70 \%$ of variance in BMI can be attributed to environment and 30\% to $40 \%$ of variance in BMI can be attributed to genetics. ${ }^{2}$ The contributions of environmental factors to the etiology of obesity are well known. Dietary choices, socioeconomic status and behavioral factors, such as inactivity, are all important factors in obese patients. Specifically, 3 Metabolic factors have been reported to be predictive of weight gain: 1) Low adjusted sedentary energy expenditure 2) High respiratory quotient (Carbohydrate- to -fat oxidation ratio and 3) Low level of spontaneous physical activity. The interaction between genetics and environment is also important. Individuals may be genetically predisposed to become obese; however, the obesity genotype may be only expressed under certain environmental conditions. In western countries, exposure to high fat diets and sedentary lifestyle is common; thus, the proportion of the population expressing the obesity genotype has increased.

\section{The Adiposity and Adipose Tissue}

Adipose tissue is composed of Lipid storing adipose cells and a stromal/ vascular component in which cells including pre adipocytes and macrophages reside. Adipose mass increases by enlargement of adipose cells through Lipid deposition, as well as by an increase in the number of adipocytes. Although the adipocyte has generally been regarded as a storage depot of fat, it is also as endocrine cell that releases numerous molecules in a regulated fashion. These molecules include the balance regulating Hormone Leptin, Cytokines such as TNF alpha and interleukin- 6, complement factors such as Factor-D (also known as adipsin), Prothrombotic agents such as plasminogen activator Inhibitor- 1 and a component of Blood Pressure regulating system- Angiotensinogen. Adiponectin, an abundant adipose derived protein whose levels are reduced in obesity, enhances insulin sensitivity and lipid oxidation and it has vascular protective effects, where as Resistin and Retinol Binding Protein (RBP4), whose levels are increased in obesity may induce insulin resistance. Leptin, the product of obgene, is a hormone secreted by adipocytes that regulates energy homeostasis and food intake via specific receptors in the hypothalamus. Most obese patients actually have elevated circulating leptin levels in the setting of functional leptin resistance, and treatment with exogenous leptin is ineffective in ameliorating the obesity. Circulating leptin concentrations are positively correlated with measures of obesity, including body mass index (BMI) and percent body fat. ${ }^{3}$

\section{Obesity and Skin Physiology}

Obesity is related to a number of effects on skin physiology, including effects on the skin barrier function, sebaceous glands and sebum production, sweat glands, lymphatics, collagen structure and function, wound healing, micro circulation and macro circulation and subcutaneous fat.

\section{Skin Manifestations of Obesity 4}

1. Insulin Resistance- Insulin resistance syndrome, acanthosis Nigricans, acrochordons, keratosis pilaris, hyperandrogenism, hirsutism.

2. Mechanical- Plantar hyperkeratosis, striae distensae, cellulite, adiposis dolorosa, lymphedema, chronic venous insufficiency.

3. Infectious- Intertrigo, candida infection, dermatophytes infection, folliculitis, necrotizing fasciitis/cellulitis.

4. Inflammatory- Hidradenitis suppurativa, psoriasis.

5. Metabolic- Tophaceous gout.

The purpose of this study is to elucidate the various skin changes occurring in obese patients, to work up the obese patients with acanthosis nigricans and skin tags for the presence of metabolic syndrome and to study their association to leptin levels and insulin levels.

\section{METHODS}

This is a cross sectional descriptive study. 50 adult patients of age group $>15$ (25 male and 25 female patients), satisfying the inclusion criteria, who attended the outpatient department of Mamatha Medical College \& Hospital, Khammam from January 2018 to January 2019. All adult patients, of both sexes, who have a BMI $>30.0$ and/or waist circumference $>90 \mathrm{cms}$ in males and $>80 \mathrm{cms}$ in females were included in the study. Patients with obesity due to congenital syndromes and age less than 15 years (pediatric age group) and non-consenting patients were excluded Patients included in the study were classified into three groups based on their BMI: class 1 with BMI 30.0-34.9, class 2 with BMI 35.0-39.9, class 3 with BMI $>40$. A detailed history of the patients were taken. Morphology of skin lesions, sites of involvement, number of lesions were noted. A complete systemic and dermatological examination was carried out in all patients. Blood pressure was evaluated. Scraping for $\mathrm{KOH}$ mount, bacterial culture done when needed. Height was recorded in meters. Weight was recorded in kilograms. Waist circumference was recorded in centimeters.

\section{Specific Blood Investigations}

- Blood sugar: Fasting blood sugar, postprandial blood sugar.

- Fasting lipid profile

- Fasting serum Insulin

- Fasting serum Leptin

\section{Interpretation of Tests}

- Body mass index was calculated according to the formula weight in $\mathrm{Kg} /$ height $^{2}$ in meters. BMI 30.0-34.9 was labeled 
as class 1 obesity, BMI 35.0-39.9 was labeled as class 2 obesity, BMI $>40$ was labeled as class 3 obesity.

- Fasting Blood sugar levels $>100 \mathrm{mg} / \mathrm{dl}$ were taken as abnormal.

- Fasting serum Insulin levels were taken as normal if present between 2.6-24.9 $\mathrm{mIU} / \mathrm{ml}$.

- Fasting serum Leptin levels were determined by an enzyme immunoassay method using Leptin ELISA kit (Manufactured by Diagnostics Biochem Canada Inc). Reference range in Males is between $2.0-5.6 \mathrm{ng} / \mathrm{ml}$ and in females is between $3.7-11.1 \mathrm{ng} / \mathrm{ml}$.

\section{Criteria for Metabolic Syndrome ${ }^{5}$}

According to the new International Diabetes Federation (IDF) definition, for a person to be defined as having the metabolic syndrome they must have central obesity i.e. waist circumference (in south Asians) in males $>90 \mathrm{~cm}$ and in females $>80 \mathrm{~cm}$, plus any two of the following four factors:

1. Raised triglycerides $>150 \mathrm{mg} / \mathrm{dl}$.

2. Reduced HDL, in men $<40 \mathrm{mg} / \mathrm{dl}$ in women $<50 \mathrm{mg} / \mathrm{dl}$.

3. Raised blood pressure $>130 / 85 \mathrm{mmHg}$.

4. Raised FBS> $100 \mathrm{mg} /$ di.

\section{Statistical Analysis}

Data was entered simultaneously into Microsoft excel worksheets designed and coded properly. The Data collected was analyzed using appropriate statistical tests, with the help of Epi-info version 3.5.1 and SPSS version 17. Chi-square and fisher exact tests have been used to test the significant association between the study parameters. A $p$ value $<0.05$ was considered to be statistically significant.

\section{RESULTS}

\begin{tabular}{|c|c|c|c|c|}
\hline \multirow{2}{*}{ Sex } & \multicolumn{3}{|c|}{ Class } & \multirow{2}{*}{ Total } \\
\cline { 2 - 4 } & Class I & Class II & Class III & \\
\hline Male & 12 & 9 & 4 & 25 \\
\hline Female & 15 & 5 & 5 & 25 \\
\hline Total & 27 & 14 & 9 & 50 \\
\hline
\end{tabular}

Table 1. Distribution of Patients Based on Sex and Class of Obesity

\begin{tabular}{|c|c|c|}
\hline Skin Condition & Frequency & Percent \\
\hline Acanthosis Nigricans & 42 & 84.0 \\
\hline Skin Tags & 38 & 76.0 \\
\hline Dermatophyte infection & 14 & 28.0 \\
\hline Fissure Feet & 13 & 26.0 \\
\hline Striae Distensae & 13 & 26.0 \\
\hline Psoriasis & 13 & 26.0 \\
\hline Pyoderma & 11 & 22.0 \\
\hline Intertrigo & 10 & 20.0 \\
\hline Varicose veins & 07 & 14.0 \\
\hline Seborrheic Dermatitis & 05 & 10.0 \\
\hline Hand-feet Eczema & 04 & 8.0 \\
\hline Stasis Eczema & 03 & 6.0 \\
\hline Table 2. Distribution of Skin Conditions among Study Patients \\
\hline \multicolumn{2}{|c}{}
\end{tabular}

\begin{tabular}{|c|c|c|c|c|}
\hline Skin Condition & Class-I & Class-II & Class-III & Total \\
\hline Acanthosis Nigricans & 19 & 14 & 9 & 42 \\
\hline Skin Tags & 16 & 13 & 9 & 38 \\
\hline Dermatophyte infection & 11 & 0 & 3 & 14 \\
\hline Fissure Feet & 3 & 7 & 3 & 13 \\
\hline Striae Distensae & 3 & 5 & 5 & 13 \\
\hline Psoriasis & 2 & 4 & 7 & 13 \\
\hline Pyoderma & 5 & 4 & 2 & 11 \\
\hline Intertrigo & 3 & 4 & 3 & 10 \\
\hline Varicose veins & 0 & 1 & 6 & 7 \\
\hline Seborrheic Dermatitis & 4 & 1 & 0 & 5 \\
\hline Hand-feet Eczema & 2 & 1 & 1 & 4 \\
\hline Stasis Eczema & 0 & 0 & 3 & 3 \\
\hline Table 3. Distribution of Skin Manifestations According to \\
Obesity-Class/BMI \\
\hline
\end{tabular}

\begin{tabular}{|c|c|c|c|}
\hline Skin Condition & Male & Female & Total \\
\hline Acanthosis Nigricans & 23 & 19 & 42 \\
\hline Skin Tags & 20 & 18 & 38 \\
\hline Dermatophyte infection & 3 & 11 & 14 \\
\hline Fissure Feet & 9 & 4 & 13 \\
\hline Striae Distensae & 5 & 8 & 13 \\
\hline Psoriasis & 5 & 8 & 13 \\
\hline Pyoderma & 9 & 2 & 11 \\
\hline Intertrigo & 7 & 3 & 10 \\
\hline Varicose veins & 3 & 4 & 7 \\
\hline Seborrheic Dermatitis & 3 & 2 & 5 \\
\hline Hand-feet Eczema & 1 & 3 & 4 \\
\hline Stasis Eczema & 1 & 2 & 3 \\
\hline Table 4. Distribution of Skin Conditions According to \\
the Sex of Study Patients \\
\hline
\end{tabular}

Table 1 demonstrates sex distribution accord to the class of obesity in class I males are $6 \%$ and females are $7.5 \%$ in class II males are $9 \%$ and females are $5 \%$ in class III males are $3 \%$ and females are $5 \%$. Table 2 illustrates distribution of skin conditions among study patients in the descending order of frequency. Acanthosis nigricans and skin tags were the most common. Table 3 demonstrates distribution of skin manifestation according to obesity class, all the skin conditions are predominant in class I obesity except psoriasis, varicose veins, stasis eczema which are predominant in class III obesity. Table 4 depicts skin conditions s per sex. Acanthosis nigricans, fissure feet, pyoderma, intertrigo were more common in obese patients.

Chi-Square Tests: The association was analyzed between the various obesity classes and Acanthosis nigricans. A p value of 0.017 shows that there was a statistically significant association between these two. $\mathrm{p}$ value: 0.010 shows significant association between the various obesity classes and skin tags. Chi-square test for linear trend: $p$ value: 0.00838 (Significant). As BMI increases, probability of patients with skin tags also significantly increases. A p value of 0.019 shows statistically significant association between obesity and striae distance. The $p$ value of 0.000 shows a statistically significant association between psoriasis and obesity. A p value of 0.000 is statistically significant which shows that there is association between obesity and varicose veins. Chi- square test for linear trend: $p$ value: 0.00003 (significant). As BMI increases, probability of patients with varicose veins also significantly increases. $p$ value of 0.001 shows significant association between obesity and stasis eczema.

\section{DISCUSSION}

In our study $48 \%$ of the male patients had grade I obesity, $36 \%$ had grade II obesity and 16 had grade III obesity. Among the female patients, $60 \%$ had grade I obesity, $20 \%$ had grade II obesity and $20 \%$ had grade III obesity.

In our study we found that Acanthosis nigricans was the most prevalent skin change in obese patients (84\%). This finding is in accordance with results of study done by Hud et al, 6 who found that $74 \%$ of obese population exhibited Acanthosis nigricans. The association between the various obesity classes and Acanthosis nigricans f significant. ( $p$ value: 0.017). As BMI increases, probability of patients with Acanthosis nigricans also significantly increases ( $\mathrm{p}$ value: 0.02167). There was no statistically significant association between Acanthosis nigricans and sex of patients. $(p=0.247)$. Skin tags was seen in $76 \%$ of study patients. Most of them had 
numerous, mixed colour, small sized skin tags. There was statistically significant association between Skin tags and BMI ( $p=0.010$ ). As BMI increases, probability of the patient to have skin tags also increases. The association between sex and Skin tag was not statistically significant. $(p=0.508)$. All of these findings are in accordance to the previous studies done by Omar Soliman el Safoury and Magdy Ibrahim. ${ }^{7}$

Out of 30 patients with both Acanthosis nigricans \& Skin tags, 19 had metabolic syndrome. Out of these 19 patients, fasting leptin levels were elevated in 17 patients. Though a greater number of patients with Acanthosis nigricans \& Skin tags with metabolic syndrome had elevated fasting leptin levels, the statistical association was found to be insignificant due to small sample size. Chi-square test $p$. value $=0.236$.)

Dermatophyte infections (tinea corporis) were present in $28 \%$ patients. There was statistically significant association between dermatophyte infection and BMI $(p=0.021)$. Though association between BMI and dermatophyte infection is significant, probability of patients to have dermatophyte infection may not increase with BMI. (Chi-square test for linear trend $\mathrm{P}$. value $=0.16044)$. There was statistically significant association between dermatophyte infection and sex $(\mathrm{p}=$ 0.0012) with more female preponderance in our study. Dermatophyte Infections were more common in diabetics when compared to non-diabetics.

Psoriasis was seen in $26 \%$ of the study patients. The association between various obesity classes and Psoriasis was statistically significant. (p value: 0.000). As BMI increases, probability of patients to have psoriasis also significantly increases ( $p$ value: 0.00014 ).These findings in accordance with the previous studies done by Sekai R et al and others. ${ }^{8}$ There was no statistically significant association between Psoriasis and sex of patients in our study. $(\mathrm{p}=0.333)$.

Striae distensae was seen in $26 \%$ of study patients. The association between the various obesity classes and striae distensae was statistically significant. ( $p$ value: 0.019 ). As BMI increases, probability of patients with striae distensae also significantly increases ( $p$ value: 0.01026). There is no statistically significant association between striae distensae and sex of patients. $(p=0.333)$. These findings are in accordance with the findings of previous studies done by J. C. Boza et al and others. ${ }^{9}$ Fissure feet was seen in $26 \%$ of study patients. The association between various obesity classes and fissure feet was statistically significant. (p value: 0.023). As BMI increases, probability of patients to have fissure feet may not significantly increase (Chi-square test for linear trend $p$ value: 0.08252 ). There was no statistically significant association between fissure feet and sex of patients. $(p=$ 0.196). These findings are in accordance with the findings of previous studies done by J. C. Boza et al and others.

Pyoderma was seen in $22 \%$ of study patients. The association between the various obesity classes and pyoderma was statistically insignificant. ( $\mathrm{p}$ value: 0.762). As BMI increases, probability of patients to have pyoderma doesn't significantly increase (Chi-square test for linear trend $p$ value: 0.83966), patients with obesity are more prone for metabolic syndrome where diabetes mellitus is one of the manifestations and which could be a cause for pyodermas. There was statistically significant association between pyoderma and sex of patients. $(p=0.017)$, seen more in males than females in our study. Pyodermas were more common in diabetics when compared to non diabetics Intertrigo was seen in $20 \%$ of study patients. In our study the association between the various obesity classes and intertrigo was statistically insignificant. ( $p$ value: 0.226 ), may be because of more maceration and friction in the flexural area. As BMI increases, probability of patients to have intertrigo may not significantly increase (Chi-square test for linear trend $p$ value: 0.15806 ). There was no statistically significant association between intertrigo and sex of patients. $(p=0.157)$. Varicose veins were seen in $14 \%$ of study patients. The association between the various obesity classes and varicose veins was statistically significant. ( $p$ value: 0.000 ), may be because of more weight bearing and gravitational impact. As BMI increases, probability of patients to have varicose veins significantly increase (Chi-square test for linear trend $p$ value: 0.00003 ). There was no statistically significant association between varicose veins and sex of patients. ( $\mathrm{p}=$ 1.000).

Seborrheic dermatitis was seen in $10 \%$ of study patients. The association between the various obesity classes and seborrheic dermatitis was statistically insignificant. ( $p$ value: 0.402). As BMI increases, probability of patients to have SD doesn't significantly increase (chi-square test for linear trend ( $p$ value: 0.10107 ). There was no statistically significant association between varicose veins and sex of patients. ( $p=$ 1.000). Hand and feet eczema were seen in $8 \%$ of study patients. The association between the various obesity classes and hand and feet eczema was statistically insignificant. ( $p$ value: 0.930 ). As BMI increases, probability of patients to have hand and feet eczema may not significantly increase (Chisquare test for linear trend $p$ value: 0.96786 ). There was no statistically significant association between varicose veins and sex of patients. $(\mathrm{p}=0.609)$.

Osteoarthritis was seen in $8 \%$ patients. The association between the various obesity classes and osteoarthritis was a statistically insignificant. ( $p$ value: 0.006 ). As BMI increases, probability of patients to have osteoarthritis significantly increase (Chi-square test for linear trend $p$ value: 0.00814 ). There was no statistically significant association between osteoarthritis and sex of patients. $(\mathrm{p}=0.110)$. Melasma was seen in $8 \%$ patients. Waist band eczema was seen in $6 \%$. Stasis eczema seen in $6 \%$. Acne vulgaris seen in $4 \%$. Seborrheic keratosis seen in $2 \%$. Lichen planus seen in $2 \% .8 \%$ had osteoarthritis.

\section{CONCLUSIONS}

Dermatologists needs to be aware of skin diseases related to obesity, so that they can advise patients for regular monitoring of blood sugars and lipid profiles. Lifestyle modifications can decrease the risk of cardiovascular and cerebro vascular accidents in obese people.

\section{REFERENCES}

[1] Fauci AS, Braunwald E, Kasper DL, et al. Harrisons Principle of Internal Medicine. 17th edn. McGraw-Hill Professional 2008: p. 462-3.

[2] Pi-Sunyer FX. The Obesity epidemic: pathophysiology and consequences of obesity. Obes Res 2002;10(Suppl 2):97S$104 \mathrm{~S}$ 
[3] Considine RV, Sinha MK, Heiman ML, et al. Serum immunoreactive-leptin concentrations in normal-weight and obese humans. N Eng Med 1996;334(5):292-5.

[4] Yoshipovitch G, DeVore A, Dawn A. Obesity and the skin: skin physiology and skin manifestations of obesity. Journal of American Academy of Dermatology 2007;56(6):901-16.

[5] Alberti KG, Zimmet P, Shaw J, et al. The metabolic syndrome - a new world wide definition. IDF Epidemiology Task Force Consensus Group. Lancet 2005;366(9491):1059-62.
[6] Hud JA Jr, Choben JB, Wagner JM, et al. Prevalence and significance of acanthosis nigricans in an adult obese population. Arch Dermatol 1992;128(7):941-4.

[7] El Safoury OS, Ibrahim M. A clinical evaluation of skin tags in relation to obesity, type 2 diabetes mellitus, age and sex. Indian Journal of Dermatol 2011;56(4):393-7.

[8] Sakai R, Matsui S, Fukushima M, et al. Prognostic factor analysis for plaque psoriasis. Dermatology 2005;211(2):103-6.

[9] Boza JC, Trindade EN, Peruzzo J, et al. Skin Manifestations of obesity: a comparative study. J Eur Acad Dermatol Venereol 2012;26(10):1220-3. 